\title{
Ictal catatonia associated with segmental catalepsy
}

\begin{tabular}{c} 
A. Bruce Janati', Naif AlGhasab², Sulaman Almesned', Mohammed Alharbi4 \\
${ }^{1}$ Center for Neurology in Fairfax Virginia, USA \\
${ }^{2}$ Department of Medicine, Medical College, Hail University, Saudi Arabia \\
${ }^{3}$ Department of Surgery, Medical College, Qassim University, Saudi Arabia \\
${ }^{4}$ Department of Medicine, Medical College, Hail University, Saudi Arabia \\
\hline
\end{tabular}

\begin{abstract}
Introduction. Non-convulsive status epilepticus (NCSE) is an epileptic condition lasting more than 30 minutes in which continuous or recurrent seizure activity on the electroencephalogram (EEG) is associated with diverse clinical symptoms including alteration of mental state, abnormal behavior, perceptual disturbances or altered consciousness. Methods. This was a case study conducted at King Khalid Hospital.

Results. Our patient presented with catatonia and segmental catalepsy associated with non-convulsive status epilepticus.

Conclusions. The simultaneous occurrence of non-convulsive status epilepticus and catatonia/catalepsy in our patient suggests a common pathophysiological mechanism. Furthermore, our data suggest that dysfunction of the posterior areas of the brain may be responsible for catalepsy.
\end{abstract}

Keywords: status epilepticus, non-convulsive, ictal catatonia, segmental catalepsy

\section{INTRODUCTION}

Non-convulsive status epilepticus (NCSE) is an epileptic condition lasting more than 30 minutes in which continuous or recurrent seizure activity on the electroencephalogram (EEG) is associated with diverse clinical symptoms including alteration of mental state, abnormal behavior, perceptual disturbances or altered consciousness [1]. The diagnosis of NCSE may be difficult due to its protean presentation [2]. This syndrome can occur in various forms of brain pathology, including stroke, brain tumors, and craniocerebral trauma, as well as in systemic diseases affecting the central nervous system [3]. EEG is considered to be the sine qua non for confirmation of the diagnosis of this condition.

In this paper we report for the first time the occurrence of segmental catalepsy in a patient with ictal catatonia associated with NCSE.

\section{CASE REPORT}

An 86-year-old Saudi male was brought to emergency room of King Khaled Hospital on July 27th, 2011 with shortness of breath and drowsiness. He had a history of an old right hemispheric hemorrhagic infarct with left hemiplegia, hypertension, hypercholesterolemia and depression. At the time of admission his medications included aspirin, simvastatin and captopril.

On examination, the patient was unresponsive with a Glasgow coma scale of $7 / 15$. Vital signs pulse: $91 /$ minute, blood pressure: $145 / 85 \mathrm{mmHg}$, temperature: $37.8 \mathrm{C}$ and RR of 20/minute. Chest examination was normal except for bilateral basal crepitation. Abdominal examination revealed abdominal distension. There was no lymph node enlargement and no skin rash. Neurological examination showed the patient to be stuporous and 
non-reactive to external stimuli. The pupils were 3 $\mathrm{mm}$ in diameter, being equal and reactive to light. There was no papilledema. Doll's eyes were present. There was no facial asymmetry. Evaluation of the motor system revealed no spontaneous movements of the extremities. A striking finding was a sustained and immobile posture of the right upper extremity with the right elbow in the flexed position at 90 degrees along with elevation of the right arm. Any attempt to change this posture would result in a rapid return of the limb to its original posture. Deep tendon reflexes were depressed all over. There were no abnormal movements. There were no meningeal signs. There were no bruits.

Laboratory investigations showed raised platelet count 479x109/1, decreased hemoglobin $(\mathrm{Hb})$ $10.5 \mathrm{~g} / \mathrm{dl}$, decreased hematocrit (Hct) $31.3 \%$, raised fasting blood glucose was $13 \mathrm{mmol} / 1$, raised serum creatinine $202 \mu \mathrm{mol} / \mathrm{l}$, raised urea level 11.14 $\mathrm{mmol} / \mathrm{l}$, decreased serum sodium $128 \mathrm{mmol} / \mathrm{l}$, decreased serum calcium $2 \mathrm{mmol} / 1$. Other laboratory investigations were within normal range.

Plain brain CT scan revealed scattered vascular calcifications in the posterior fossa. There were right parietal lobe, right basal ganglia, left frontal lobe and left parietal lobe low density areas compatible with multiple subacute to chronic infarcts, being more prominent on the left. There was no midline shift. Brain MRI showed old infarct in the left middle cerebral artery distribution with a prominent left ventricle. There was a right paraventricular subacute infarct with effacement of the right lateral ventricle. There was diffuse cortical atrophy with old white matter ischemic changes. A portable EEG was consistent with non-convulsive status epilepticus (figure 1 and 2).

A carotid Doppler study revealed bilateral intimal thickening of both carotid arteries and its branches. No evidence of calcified plaques or significant stenosis. Echocardiogram revealed an ejection fraction of $60 \%$ and left ventricular hypertrophy without thrombi.

In the emergency room, he received Midazolam $10 \mathrm{mg}$, Fentanyl $1 \mathrm{mg}$ for intubation, Acetaminophen $1 \mathrm{~g} / 100 \mathrm{ml}$ and Phenytoin $300 \mathrm{mg}$ were given. The patient underwent rapid sequence intubation and was placed on mechanical ventilator and was later admitted to the intensive care unit (ICU) where a fever work-up was negative. The patient's condition remained unchanged. He died of cardiopulmonary arrest.

\section{DISCUSSION}

Catatonia is a syndrome characterized by a psychomotor disturbance. Two types mentioned in the literature: retarded and excited. Catatonia with retardation is associated with signs reflecting a paucity of motor movements, including immobility, staring, mutism etc. (4).

Catatonia with excitement is characterized by psychomotor agitation including hyperthermia, altered mental status, and autonomic dysfunction which is referred to as malignant catatonia [4].

The DSM-V defines catatonia as the presence of three or more of the following: catalepsy, waxy flexibility, stupor, agitation, mutism, negativism, posturing, mannerisms, stereotypies, grimacing, echolalia, and echopraxia. A number of scales have been developed to quantify catatonic signs [5].

Our patient presented with stupor, motor immobility, and lack of response to external stimuli, consistent with catatonia. An EEG confirmed the diagnosis of NCSE which in this particular case, most likely emanated from an old left hemispheric infarct. A striking finding was the presence of "segmental catalepsy" manifested by a sustained, rigid, and immobile posture of the right upper extremity which could be overcome by application of external force, however, with a rapid return of the limb to the original posture (flexibilitas cerea). The catalepsy seemed to correspond to the focal electrographic seizure involving the contralateral occipital-posterior temporal region.

It has been suggested that hypoactivity of GABA (the primary inhibitory neurotransmitter of the brain) in the corticostriatal pathways or hyperactivity of glutamate (the primary excitatory neurotransmitter) may be the underlying pathophysiological mechanisms of catatonia [6]. On the other hand, similar mechanisms have been implicated in the pathogenesis of status epilepticus [7]. The simultaneous occurrence of both syndromes in our patient is concordant with this analogy and suggests a common pathophysiological mechanism. Furthermore, the presence of segmental catalepsy as a clinical manifestation of the contralateral focal electrographic seizure is additional evidence in support of 


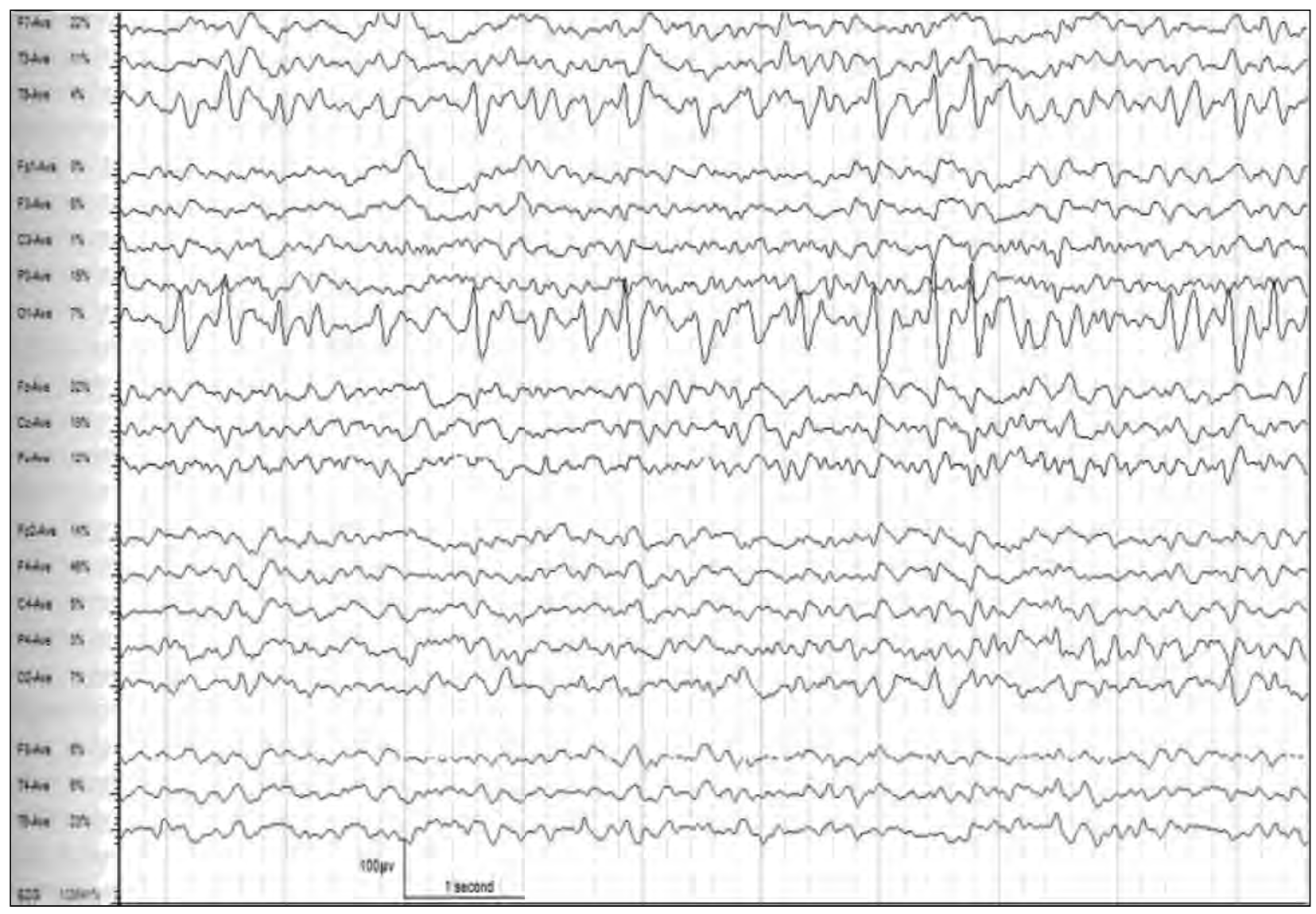

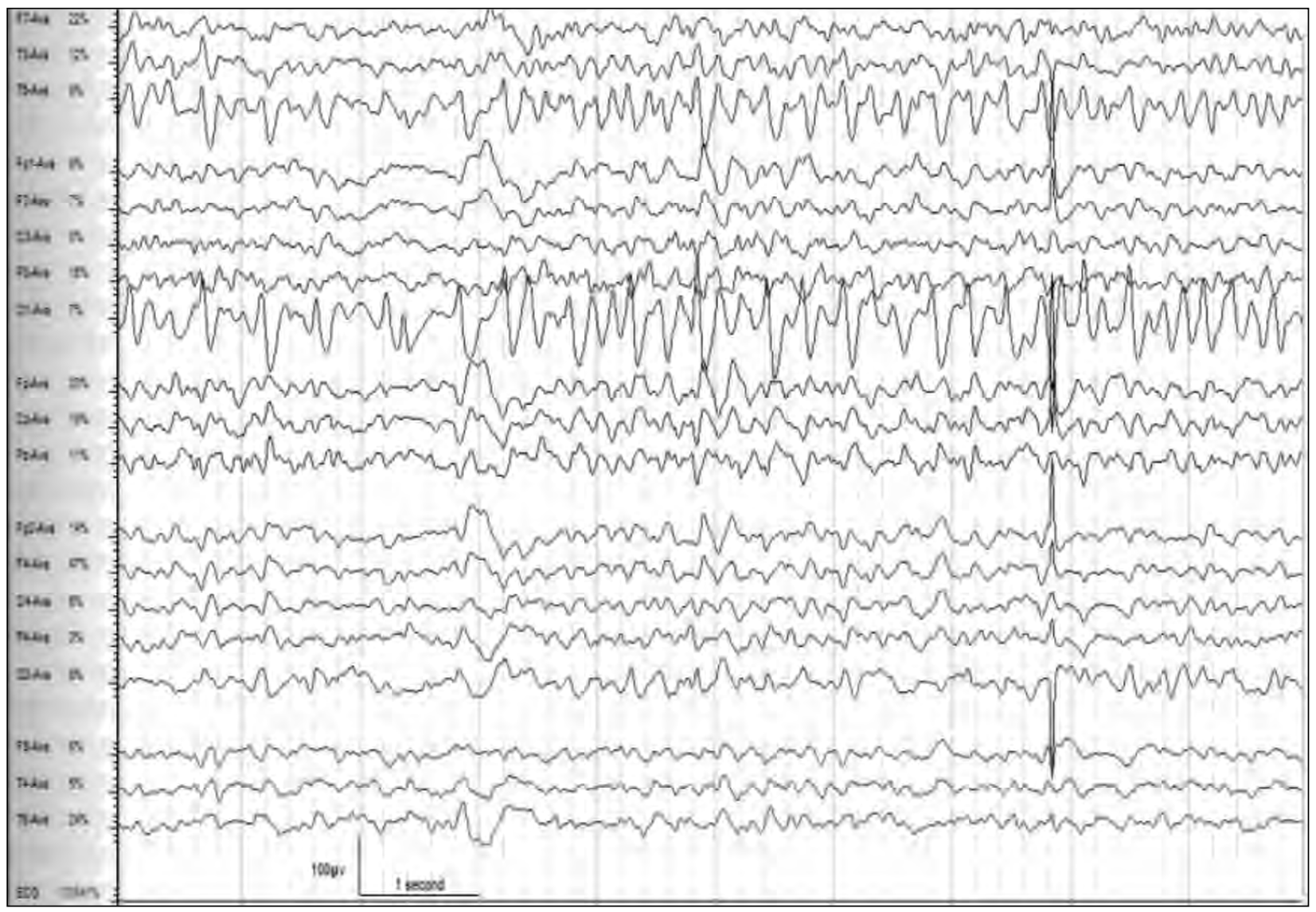


this theory. However, it is intriguing that this electrical activity only involved the left occipital-posterior temporal region, raising the possibility that dysfunction of the posterior areas of the brain may be responsible for this motor abnormality (catalepsy). Further studies are needed to confirm this hypothesis.

Conflict of interest: none declared

Financial support: none declared

\section{REFERENCES}

1. Fernández-Torre JL, Gutiérrez-Pérez R, Velasco-Zarzosa M. Non-convulsive status epilepticus. Revista de Neurología 2003;37(8):744-52.

2. Rüegg S. Non-convulsive status epilepticus inadults - an overview. Schweiz Arch Neurol Psychiatr. 2008;159:53-83.

3. Jankowicz E, Drozdowski W, Halicka D. "De novo" non-convulsive status epilepticus in adults and in the elderly. Neurol Neurochir Pol. 2003;37(3):621-32.

4. Sean R, Michael F, Patricia IR. Catatonia: Our current understanding of its diagnosis, treatment and pathophysiology. 2016 Dec 22. doi: 10.5498/wjp.v6.i4.391

5. Rasmussen SA, Mazurek MF, Rosebush PI. Catatonia: Our current understanding of its diagnosis, treatment and pathophysiology. World J Psychiatry. 2016;6(4):391-398.

\section{CONCLUSIONS}

The simultaneous occurrence of non-convulsive status epilepticus and catatonia/catalepsy in our patient suggests a common pathophysiological mechanism. Furthermore, our data suggest that dysfunction of the posterior areas of the brain may be responsible for catalepsy.

6. Tandon R, Heckers S, Bustillo J, Barch DM, Gaebel W, Gur RE, Malaspina D, Owen MJ, Schultz S, Tsuang M, et al. Catatonia in DSM-5. Schizophr Res. 2013;150:26-30.

7. Northoff G, Eckert J, Fritze J. Glutamatergic dysfunction in catatonia? Successful treatment of three akinetic catatonic patients with the NMDA antagonist amantadine. Journal of Neurology, Neurosurgery and Psychiatry. 1997;62:404-406.

8. ACF Hui, Cyman, HC Wong. Management of Status Epilepticus. Hong Kong Journal of Emergency Medicine. 2002;9:206-212. 